\title{
A flow-induced phase inversion in immiscible polymer blends containing a liquid-crystalline polymer studied by in situ optical microscopy
}

\author{
Marianne Astruc and Patrick Navard ${ }^{\text {a) }}$ \\ Ecole des Mines de Paris, Centre de Mise en Forme des Matériaux, \\ UMR CNRS 7635, BP 207, 06904 Sophia Antipolis Cedex, France
}

(Received 13 August 1999; final revision received 27 March 2000)

\section{Synopsis}

The phase inversion from a morphology of hydroxypropylcellulose in water (HPC50\%) droplets in polydimethylsiloxane (PDMS) matrix to a morphology of PDMS droplets in HPC50\% matrix can be induced by a change of shear rate, due to a viscosity ratio inversion. Such a process passing through four different transient morphological stages was studied by optical microscopy in a transparent shear device. In a certain concentration region, at a fixe shear rate, after sheets of PDMS were formed, the transition "hesitates" between phase inversion and refine starting morphology. The influenc of PDMS concentration, shear rate, elasticity, and phase dimension on the fina morphology was investigated and compared with different models given in the literature. The influenc of concentration and shear rate on the duration of the transient flo was also studied. We propose a simplifie model of breakage of filament to explain a part of our results. (C) 2000 The Society of Rheology. [S0148-6055(00)01504-2]

\section{INTRODUCTION}

The increasing application of polymer blends for the elaboration of new materials is motivating the general interest in the study of morphology development in immiscible polymer blends, since morphology is one of the major factors controlling their fina properties. For two-phase immiscible polymer blends, different morphologies such as droplet, fibrilla or co-continuous structures can be found. Several parameters are important in determining the fina or steady-state morphology: composition of the blend, shear rate, viscosity and elasticity of the two phases, interfacial tension, and time of mixing. Among the many morphological phenomena that occur during preparation and processing of polymer blends, the study and prediction of the point of phase inversion at which co-continuity is observed has attracted a lot of attention. All the published studies are based on semiempirical models.

Some authors have related the point of phase inversion with the rheological properties of the components. Avgeropoulos et al. (1976) suggested that the point of co-continuity is reached when the torque ratio of the components in an internal mixer is equal to the component volume fraction ratio. Later, Paul and Barlow (1980) proposed a similar formulation using the viscosity ratio instead of the torque ratio. Miles and Zurek (1988) proposed the following generalized expression: 


$$
\frac{\Phi_{A} \eta_{B}(\dot{\gamma})}{\Phi_{B} \eta_{A}(\dot{\gamma})}\left\{\begin{array}{l}
>1: \text { phase } A \text { is continuous } \\
=1: \text { co-continuity } \\
<1: \text { phase } B \text { is continuous }
\end{array}\right.
$$

where $\Phi_{A}$ and $\Phi_{B}$ are the volume fractions of the phases $A$ and $B$, and $\eta_{A}$ and $\eta_{B}$ their viscosities at the applied shear rate $\dot{\gamma}$.

Several other models predicting the point of phase inversion are based on the filamen instability concept. Metelkin and Blekht (1984) considered the dispersed phase to be that with the shorter breakage time of filaments The critical volume fraction for the phase inversion was calculated to be

$$
\Phi_{B}=\frac{1}{1+\lambda F(\lambda)}
$$

where

$$
F(\lambda)=1+2.25 \log \lambda+1.81(\log \lambda)^{2},
$$

with $\lambda$ being the viscosity ratio of the blend components at the blending shear rate. Luciani et al. (1993) made the assumption that there is a two-fibrilla network structure at the co-continuity point. They supposed that the threads have the same length and stability at the phase inversion point, so that

$$
\left(\frac{\Phi_{B}}{\Phi_{A}}\right)^{0.5}=\frac{\eta_{A} \Omega\left(\frac{\eta_{B}}{\eta_{A}}\right)}{\eta_{B} \Omega\left(\frac{\eta_{A}}{\eta_{B}}\right)}
$$

where $\Omega(\lambda)$ is a function tabulated by Chappelear (1964).

Utracki (1991) suggested a model that can be used to predict the point of phase inversion for blends with a viscosity ratio far from unity. It is based on the dependence of the viscosity on the volume fraction of monodispersed hard spheres in the matrix, as proposed by Krieger and Dougherty (1959). The point of co-continuity is where the viscosity of polymer $A$ (at the volume fraction $\Phi_{A}$ ) dispersed in polymer $B$ equals the viscosity of polymer $B$ (at the volume fraction $\Phi_{B}$ ) in polymer $A$. At this point, the ratio is

$$
\lambda=\left[\frac{\left(\Phi_{m}-\Phi_{B}\right)}{\left(\Phi_{m}-\Phi_{A}\right)}\right]^{[\eta] \Phi_{m}},
$$

where $[\eta]$ is an intrinsic viscosity and $\Phi_{m}=1-\Phi_{c}$ is the maximum packing volume fraction. $\Phi_{c}$ is the critical volume fraction for percolation, equal to 0.156 for threedimensional spherical dispersions [Chapoy (1986)]. An arbitrary value of $[\eta]=1.9$ was given by Utracki (1991), based on published data on the points of phase inversion of thermoplastics and rubbers.

The model of Avgeropoulos predicts satisfactorily the point of phase inversion for torque ratios close to unity. The model of Miles and Zurek (1) and the model of Metelkin and Blekht (2) were found to overestimate the composition at which phase inversion occurs if the viscosity ratio departs from unity while the model of Utracki (5) was found to agree with some experimental results for blends having a viscosity ratio far from unity [Utracki (1991), Mekhilef and Verhoogt (1996), Mekhilef and Verhoogt (1997)]. The 
agreement of these models with experiments is strongly dependent on the nature of the blend under consideration, underlining the imperfection of these models. The failure of the models may be due to the fact that the elasticity of each component polymer or the duration of the blending process are not taken into account. More recently, other models where the elasticity or the interfacial tension are considered have been established. Because the more elastic phase will tend to encapsulate the less elastic one and form the matrix, Bourry and Favis (1998) proposed the following expression for the point of co-continuity:

$$
\frac{\Phi_{A}}{\Phi_{B}}=\frac{G_{B}^{\prime}}{G_{A}^{\prime}} \cdot \frac{G_{A}^{\prime \prime}}{G_{B}^{\prime \prime}},
$$

where $G_{i}^{\prime}$ and $G_{i}^{\prime \prime}$ are, respectively, the storage and the loss modulus of the component $i$. This expression seems to give better results than expressions (1), (2), and (5) when the elasticities of the two components differ strongly. By considering a co-continuous structure as a dense packing of randomly oriented rodlike particles of the minor phase and taking into account the microrheological requirements for the stability of such rods, Willemse et al. (1998) established an equation predicting the range of compositions within which fully co-continuous structures can exist. The equation relates the critical volume fraction of the minor phase at complete co-continuity $\Phi_{d, \mathrm{cc}}$ to the matrix viscosity $\eta_{m}$, interfacial tension $\sigma$, shear rate $\dot{\gamma}$ and phase dimension $R_{0}$ :

$$
\frac{1}{\Phi_{d, \mathrm{cc}}}=1.38+0.0213\left(\frac{\eta_{m} \dot{\gamma}}{\sigma} R_{0}\right)^{4.2} .
$$

An absolute prediction based on this model is not straightforward because the phase dimension has to be measured (or even worse, has to be define for a real blend). The authors have experimentally shown that $\Phi_{d \text {,cc }}$ is slightly influence by $\eta_{m}$ [Willemse et al. (1998)] whereas an increase of $\sigma$ leads to an increase of $\Phi_{d, \mathrm{cc}}$ and a decrease of the range of co-continuity [Willemse et al. (1999a)].

Flow-induced phase inversion occurs when the original morphology of polymer $A$ dispersed in the matrix of polymer $B$ is transformed under the action of a flo into polymer $B$ dispersed in polymer $A$. Clearly, this must involve crossing the point of co-continuity. Such crossings have been observed after blend extrusion [Shih (1992)] and studied in batch mixers [Shih (1995), Ratnagiri and Scott (1996)]. In some cases, the observations could be explained by differences in the melting temperature of the components (i.e., phase $A$ supposed to be the matrix is molten at a higher temperature than phase $B$, which gives solid $A$ in molten $B$, and which inverses when $A$ melts). But it appears clearly that in most cases, a variation of viscosity or elasticity during the blending process plays a major role [Ratnagiri and Scott (1996), Ratnagiri and Scott (1997)]. The main problem is that observations after extractions do not allow us to clearly know how co-continuity is reached during the flo and how the morphologies change around that point. This lack of knowledge makes it difficul to build a comprehensive model. To our knowledge, no in situ experiment on this effect has been reported.

Using the torque or viscosity ratio concept, one may understand qualitatively how a phase inversion can be induced by the flow By varying the shear rate, the viscosity ratio may vary. If this variation is large enough, it could induce a phase inversion [Eqs. (1), (4) or (5), depending on the model]. Bouilloux et al. (1997) studied a phase inversion induced by the variation of viscosity ratio with time of mixing. By quenching the blend at various stages of mixing, they always observed the co-continuous structure. They could not reach the inverted morphology. Willemse et al. (1999a) assumed that sheets of the 
initial dispersed phase could be the precursors of the co-continuous morphology. Depending on the stability of the network of ligaments in the mixer after sheet breakup, the co-continuous morphology could be either a transient or a stable morphology. The formation and the stability of these kinds of sheets under a simple shear flo in viscoelastic blends was firs reported by Sundararaj et al. (1995). More recently, Lazo and Scott (1999) confirme with extraction experiments on PS/PE blends that phase inversion in steady-shear flo pass through sheet formation. Sheet formation that transforms into threads or drops seems to be an important factor in polymer blend deformation [Willemse et al. (1999b)].

The objectives of the present work are to use rheo-optical tools to visualize a shearinduced phase inversion in an immiscible polymer blend, to study how it occurs, and to compare this to the theories that are available. For reasons that will be detailed later, we used a blend of PDMS and a solution of hydroxypropylcellulose in water (HPC50\%). One of the difficultie in studying phase inversion and how it occurs is that the point of phase inversion is classically reached by increasing concentration, which implies changing samples. The advantage of the phase inversion observed here is that the cocontinuous morphology should be a transient state that takes place between two dropletmatrix morphologies, in the same sample. This opens the way to study two related phenomena, i.e., the mechanisms controlling both the co-continuity and the phase inversion.

\section{EXPERIMENTAL SETUP AND PREPARATION PROCEDURES}

\section{A. Rheo-optical tools}

Flow-induced morphology observations were performed on a rheo-optical system comprising three main parts:

(i) A Linkam CSS 450 shearing system. It is composed of two transparent rotating parallel plates permitting the observation of the sample at $7.5 \mathrm{~mm}$ from the center axis of rotation. The shearing system is monitored by a PC using the LINKSYS 1.41 software.

(ii) An optical system composed of an optical microscope Leitz Metallux 3 with a lighting system, a charge-coupled device camera, and a monitor.

(iii) A video data acquisition system (video recorder and time code generator).

\section{Experimental difficulties}

From a practical point of view, the study of phase inversion mechanisms with rheooptical tools is not simple. The formation of co-continuous structures in polymer blends requires a relatively high concentration of both components. But the observation of morphology in concentrated blends is difficult concentrated blends are highly scattering and so turbidity limits observation of their morphology by optical microscopy. That is why it was chosen to shear thin layers (a gap between 50 and $100 \mu \mathrm{m}$ ). We are aware of the fact that this restriction could influenc the morphologies. But this is the only way to perform in situ observations with such highly concentrated systems. In the experiments special case was taken to ensure that the observations are not significantl modifie by the small dimensions of the gap.

Furthermore, due to the complex morphologies that were formed, it is difficul to identify each phase and to recognize which polymer is where. A way to overcome this is to label one of the polymers. By choosing an isotropic component and a birefringent one, it is possible to distinguish the two phases between crossed polarizers. Unfortunately, our 
blends are liquid at room temperature and do not allow us to carry out extraction experiments after the shear experiments.

\section{B. Selected materials}

Materials with the required rheological and optical properties were selected. All experiments were performed on blends of PDMS from Rhône-Poulenc (Rhodorsil 47V200000, $\overline{M_{W}}=149350$ ) and a solution of hydroxypropylcellulose diluted by 50 wt $\%$ in water (HPC50\%). The HPC is produced by Aqualon (Klucel L, $M_{W}$ $=100000$ ). These components are liquid at room temperature. To avoid problems of phase separation that have been reported for the solution of $50 \%$ of HPC in water [Guido and Grizzuti (1995)], the experiments were performed at $18^{\circ} \mathrm{C}$. Under these conditions, the solution of HPC is in a liquid-crystalline phase. It is birefringent, whereas the PDMS is not. It is thus possible to distinguish the two components by using depolarized light microscopy. We take a literature value of the interfacial tension equal to $12.2 \mathrm{mN} / \mathrm{m}$ as reported by Tsakalos [Tsakalos (1995)] as obtained for $50 \mathrm{wt} \%$ HPC (Klucel L) in a PDMS (Rhodorsil) matrix.

\section{Rheology}

The steady shear viscosity measurements of the pure components were carried out at $18^{\circ} \mathrm{C}$ with a controlled stress Stresstech Rheologica rheometer, in parallel plate geometry ( $1 \mathrm{~mm}$ gap and $25 \mathrm{~mm}$ diameter). Shear rates from 0.1 to $20 \mathrm{~s}^{-1}$ were investigated. The measurements performed on the HPC solution were made after sufficientl large deformations to reach the steady-state conditions in lyotropic LCPs [Moldenaers (1987)]. Viscosity data of the two components are presented in Fig. 1(a). The zero shear viscosity of the PDMS is $240 \mathrm{Pas}$ compared with $480 \mathrm{Pas}$ at $0.1 \mathrm{~s}^{-1}$ for HPC50\%. Below $8 \mathrm{~s}^{-1}$, the PDMS is Newtonian while the HPC50\% solution presents a strong shear thinning behavior. The viscosity curves cross at a shear rate of $0.8 \mathrm{~s}^{-1}$. Below this shear rate, the PDMS is the least viscous component while above it is the most viscous. Over the shear rate range under investigation $\left(0.1-20 \mathrm{~s}^{-1}\right)$, the viscosity ratio of the components can easily be changed by varying the shear rate. Different viscosity ratios depending on the

shear rate are specifie in Table I. By changing the shear rate from 0.2 to $10 \mathrm{~s}^{-1}$, the viscosity ratio varies from 1.7 to 0.4 .

First normal stress difference $N_{1}$ data are plotted in Fig. 1(b). Below $12 \mathrm{~s}^{-1}$, the solution of HPC $50 \%$ has the highest elasticity. This is confirme by the measurements of the storage and loss moduli carried out at a stress of $100 \mathrm{~Pa}$. At low frequencies, the solution of HPC $50 \%$ has the highest elasticity while the loss modulus is the lowest (Fig. 2). The storage moduli cross at $11 \mathrm{rads}^{-1}$ and the loss moduli at $0.30 \mathrm{rad} \mathrm{s}^{-1}$, close to the values found in steady-state experiments. Even if the Cox-Merz rule is not strictly verifie for the HPC solution, these results show that dynamics measurements allow us to compare the relative elasticity of both components, especially for the higher shear rates that cannot be reached in continuous measurements.

\section{Preparation of the blends}

Preparation of the solutions of HPC was made following the method described by Tsakalos (1995). To avoid possible diffusion of water in PDMS that could change the HPC concentration in the HPC50\% component, the PDMS was saturated with water before blending [Tsakalos (1995)].

In order to study phase a inversion induced by a change in the viscosity ratio, it was necessary to prepare blends for which the PDMS is the continuous phase at rest. During 
a)

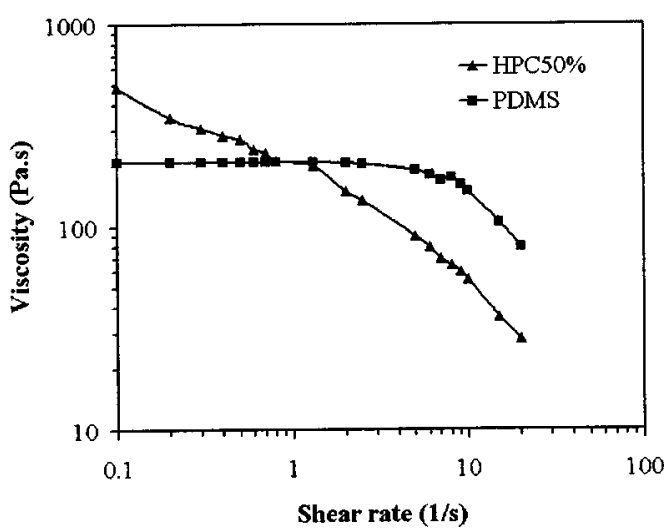

b)

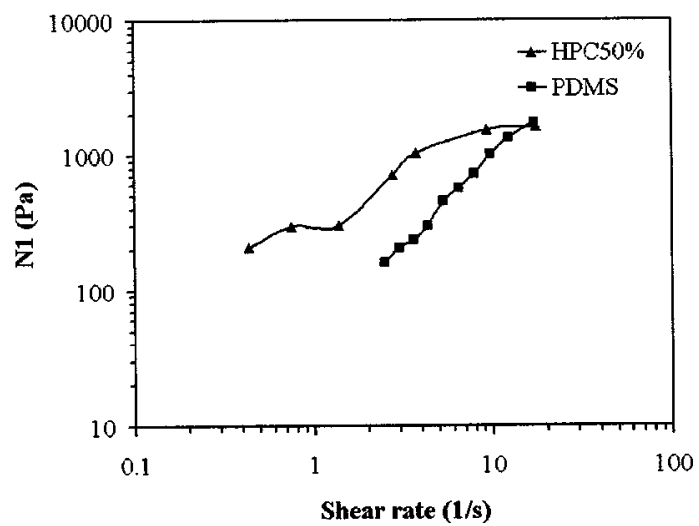

FIG. 1. Shear viscosity (a) and $N_{1}$ (b) of PDMS and $\mathrm{HPC} 50 \%$ measured at $18{ }^{\circ} \mathrm{C}$ in a plate-plate geometry.

this blending procedure, special care was taken not to cross the point of phase inversion. This means that the samples had to be submitted to very low shear rates (under $2 \mathrm{~s}^{-1}$ ). The blends were hand mixed after cooling in a refrigerator. Suitable amounts of the components were weighed and mixed very slowly for $\sim 10$ min with a spatula until a homogeneous creamy appearance was obtained.

For the preparation, two opposite points have to be balanced. On one side, the blends have to be mixed during sufficientl long time and with sufficientl high speed of rotation to give a good homogenization and stability. On the other hand, for high concentrations of the dispersed phase, the point of phase inversion is easily crossed under these conditions. A phase separation is observed after a few hours and it is also difficul to reproduce the same initial morphology for different samples with the same concentrations. Blends of 70, 67, 63, 60, and 55 vol. \% of PDMS were prepared by this procedure. They are, respectively, referred to PDMS70, PDMS67, PDMS63, PDMS60, and PDMS55. We were not able to prepare blends with a lower concentration of PDMS where PDMS was the continuous phase at rest.

\section{Experimental conditions}

Startup experiments in the Linkam shearing system were carried out at different shear rates $\left(0.8-30 \mathrm{~s}^{-1}\right)$ and concentrations $(55 \%-70 \%$ of PDMS). The indicated shear rates correspond to the shear rates applied at the location of observation in plane-plane ge- 
TABLE I. Viscosity ratio and storage modulus ratio of the HPC $50 \%$ and the PDMS at $18^{\circ} \mathrm{C}$ calculated at constant shear rate.

\begin{tabular}{ccc}
\hline \hline $\begin{array}{c}\text { Shear rate } \\
(1 / s)\end{array}$ & $\eta_{\text {HPC } 50 \%} / \eta_{\text {PDMS }}$ & $G_{\text {HPC } 5 \%}^{\prime} / G_{\text {PDMS }}^{\prime}$ \\
\hline $1.5 \times 10^{-3}$ & 7.3 & - \\
0.2 & 1.7 & 40 \\
0.5 & 1.3 & 11 \\
0.8 & 1 & 9.8 \\
2.5 & 0.7 & 3.1 \\
10 & 0.4 & 1 \\
15 & 0.4 & 0.8 \\
20 & 0.4 & 0.7 \\
30 & $0.4^{\mathrm{b}}$ & 0.5 \\
\hline \hline
\end{tabular}

assuming that the Cox-Merz rule is verified

${ }^{b}$ Value extrapolated from Fig. 1(a).

ometry, given the thickness of the sample and speed of rotation. All the experiments were done at $\pm 8 \%$ of the indicated shear rates. These variations are associated with the radial gradient of the shear rate throughout the width of visualization. First, the transient flo for blends with concentration of PDMS varying from $55 \%$ to $70 \%$ was studied at a startup of $10 \mathrm{~s}^{-1}$. Second, the transient flo of PDMS63 was studied under different startup experiments with shear rates varying from 0.8 to $30 \mathrm{~s}^{-1}$. The lower limit of the range of concentrations is limited by the difficultie of preparation described in the preceding section (phase inversion may occur during the blending procedure). The highest value of shear rate that can be used is $30 \mathrm{~s}^{-1}$, above which the blend is expulsed from the shearing device after few minutes of shearing.

In Sec. III, the transient flo observed for the blends at a shear rate of $10 \mathrm{~s}^{-1}$ will be presented in detail. These results illustrate the typical mechanisms occurring during a well-controlled but highly specifi flo induced phase inversion.

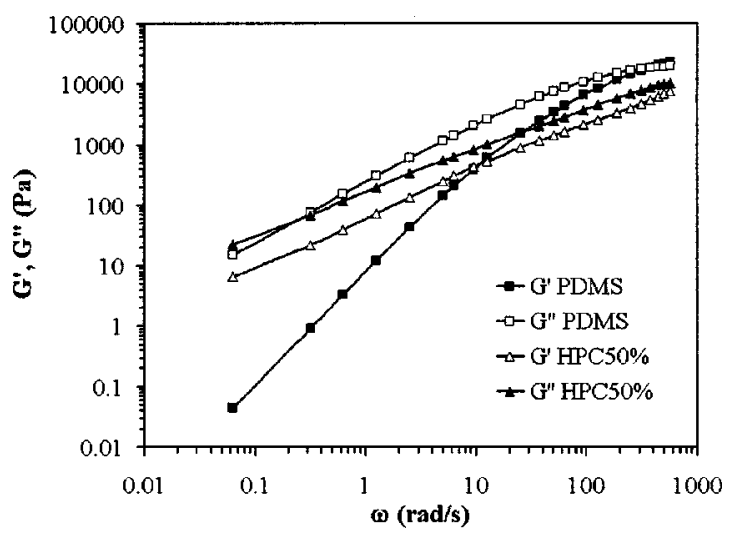

FIG. 2. Storage and loss modulus data of PDMS and HPC $50 \%$ measured at $18{ }^{\circ} \mathrm{C}$ in a cone and plate geometry. 
a)

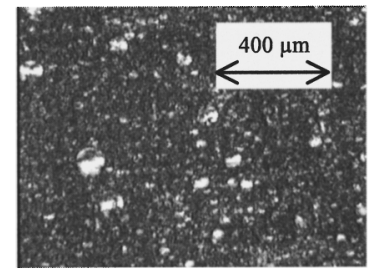

b)

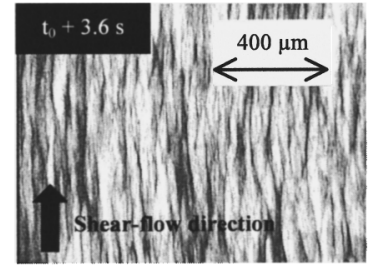

c)

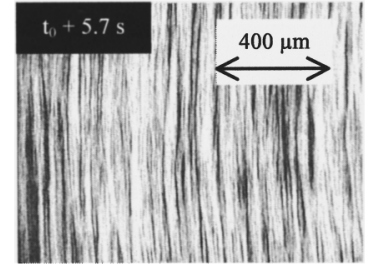

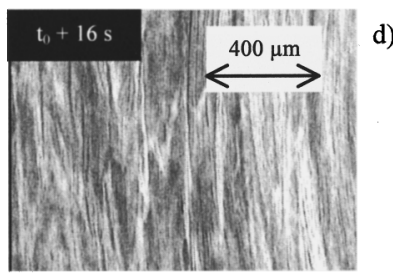
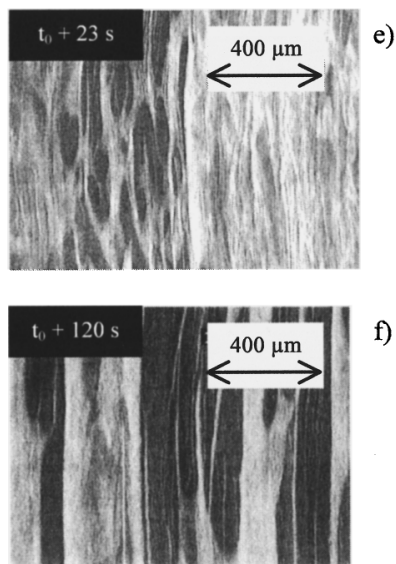

FIG. 3. The different morphologies of the blends PDMS/HPC50\% observed between crossed polarizers in the Linkam device at $18{ }^{\circ} \mathrm{C}$. The PDMS appears black and the HPC50\% bright. (a) At-rest morphology of the blend PDMS60. (b) and (c) Morphology of the blend PDMS67 during the firs stage of shearing at $10 \mathrm{~s}^{-1}$. (d) and (e) Morphology of the blend PDMS60 and (f) the blend PDMS67 during the second stage of shearing at $10 \mathrm{~s}^{-1}$.

\section{RESULTS AND DISCUSSION}

\section{A. Observed morphologies}

\section{At-rest morphology}

After carefully and slowly fillin the Linkam shearing system with a small amount of blend and adjusting the gap, the sample was allowed to relax and to reach a temperature of $18^{\circ} \mathrm{C}$ for $30 \mathrm{~min}$. For the concentrations of PDMS from $55 \%$ to $70 \%$, similar morphologies were observed [Fig. 3(a)]; droplets of HPC 50\% of diameter under $100 \mu \mathrm{m}$ are dispersed in the matrix of PDMS. Some droplets contain small subinclusions of PDMS. Favis and Chalifoux (1988) and Bourry and Favis (1998) reported the presence of similar encapsulations in blends of polypropylene/polycarbonate and in blends of polyethylene/ polystyrene around the point of phase inversion. In our blends, we attribute the presence of these encapsulations in the at-rest morphology to the fact that some local phase inversion may have occurred during the blending preparation.

\section{Transient morphologies (example of shear at $10 \mathrm{~s}^{-1}$ )}

Submitted to a simple shear flo at a shear rate of $10 \mathrm{~s}^{-1}$, the blend is in a region where a phase inversion is expected to take place. Several different transient morphologies were observed before the fina steady-state equilibrium morphology was reached. Four different stages in the mechanism leading to the fina equilibrium can be distinguished. These four stages occurred in all the observed blends and can be considered as the general route that the PDMS/HPC50\% blends take toward phase inversion. 
a)

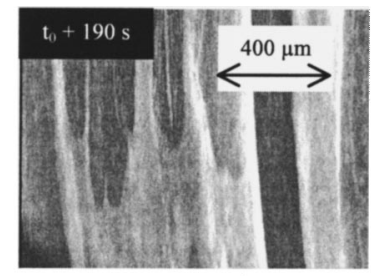

b)

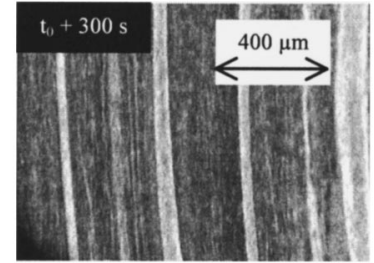

c)

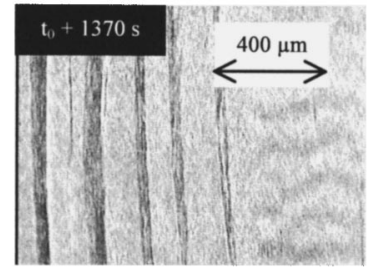

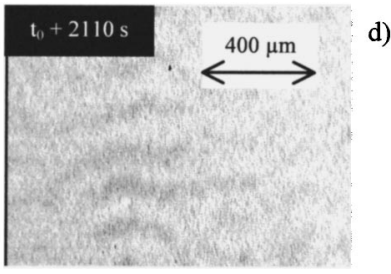

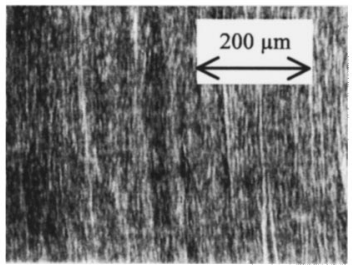

e)

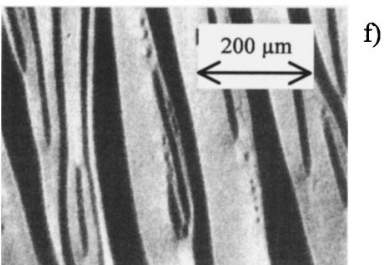

FIG. 4. In the blend PDMS60: (a) the large domains of PDMS are stretched along the flo direction; (b) stripes of continuous phases of PDMS and continuous phases of HPC50\% are formed; (c) the stripes of PDMS become thinner. Filaments originating from these stripes are dispersed in the continuous phase of HPC50\%; (d) The stripes of PDMS have completely disappeared and the phase inversion has propagated through the whole sample. We can observe a continuous phase of HPC $50 \%$ with droplets of PDMS inside. This is case (b) of Fig. 5. (e) In PDMS67: at the end of the fourth stage, the stripes of local phase inversion have disappeared: the flow-induce phase inversion does not occur and the fina morphology consists of droplets of HPC $50 \%$ in the continuous phase of PDMS, as shearing. The difference is the much smaller size of the HPC $50 \%$ droplets [see Fig. 3(a)]. This is case (c) of Fig. 5. (f) PDMS67 sheared $15 \mathrm{~h}$ at $0.2 \mathrm{~s}^{-1}$ after phase inversion.

First stage $\left(t_{0} \rightarrow t_{0}+10 \mathrm{~s}\right.$ at $\left.10 \mathrm{~s}^{-1}\right)$ : bi-fibrilla morphology. During the firs $10 \mathrm{~s}$ of shearing at $10 \mathrm{~s}-1$, the inclusions of HPC $50 \%$ and the subinclusions of PDMS are deformed and stretched along the flo direction [Fig. 3(b)]. Soon after, it is difficul to tell which phase is the continuous one. The PDMS and the HPC50\% phases appear like long filaments and the morphology can be described as a bifibrilla structure [Fig. 3(c)].

Second stage $\left(t_{0}+10 \mathrm{~s} \rightarrow t_{0}+60 \mathrm{~s}\right.$ at $\left.10 \mathrm{~s}^{-1}\right)$ : emergence of sheets of PDMS. The bifibrilla morphology is not stable. The shorter filament of PDMS begin to break [Fig. 3(d)] while at the same time, sheets of PDMS layered in the flo direction form [Fig. $3(\mathrm{e})]$. These domains of PDMS contain filament and droplets of $\mathrm{HPC} 50 \%$ with small subinclusions of PDMS. They are dispersed in a continuous phase of HPC50\% also containing small droplets stemming from the shorter filament of PDMS [Fig. 3(f)].

Third stage $\left(t_{0}+60 \mathrm{~s} \rightarrow t_{0}+600 \mathrm{~s}\right.$ at $\left.10 \mathrm{~s}^{-1}\right)$ : stripe morphology. For a longer shearing time, the sheets of PDMS continue to stretch along the flo direction [Fig. 4(a)] until they form stripes: continuous phases of HPC $50 \%$ with small droplets of PDMS dispersed alternately with continuous phases of PDMS containing inclusions of HPC $50 \%$ with subinclusions of PDMS [Fig. 4(b)]. In fact, we can consider that by this stage, a phase inversion has already occurred in the stripes of HPC $50 \%$, between the stripes of PDMS.

Fourth stage $\left(t_{0}+600 \mathrm{~s} \rightarrow t_{0}+10000 \mathrm{~s}\right.$ at $\left.10 \mathrm{~s}^{-1}\right)$ : homogenization of the morpholo- 
a)

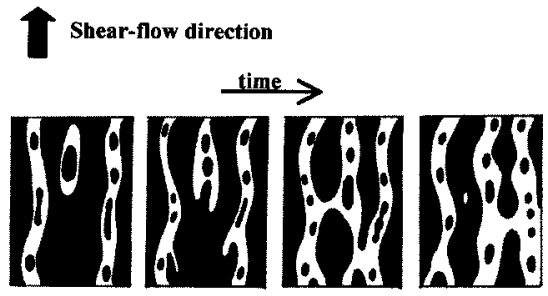

Network of $\mathrm{HPC} 50 \%$ threads (white) in the PDMS matrix (black)

b)

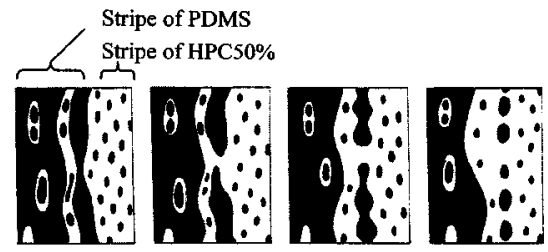

Mechanism for phase

inversion ( $\mathrm{HPC} 50 \%$

becomes the matrix)

c)

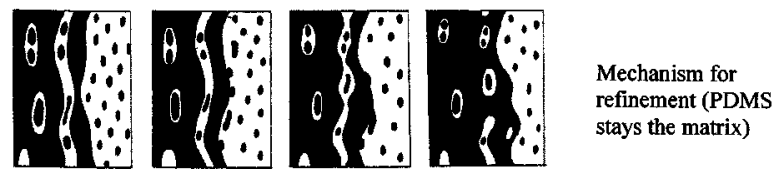

FIG. 5. (a) Schematic representation of the network of threads of HPC $50 \%$ in the stripes where the PDMS is the continuous phase observed during the fourth stage. (b) Schematic representation of the mechanisms observed at the edge of the stripes during the fourth stage when a flament of $\mathrm{HPC} 50 \%$ is close to the edge: the edge of PDMS breaks up and the stripe of HPC $50 \%$ enlarges. (c) The flament of HPC $50 \%$ breaks up and the stripe of HPC $50 \%$ thins.

gy. After the stripes have been formed, the morphology evolves extremely slowly. Different mechanisms occur independently in different zones in the blend.

(i) In the stripes where the PDMS is the continuous phase, we observe long filament of HPC $50 \%$ constituting a kind of relatively unstable network in which branches are continuously forming and breaking. Some of these filament break to form smaller droplets. These small inclusions coalesce to reform filaments Inside the inclusions of HPC $50 \%$, the subinclusions of PDMS are also subject to the mechanisms of breakup and coalescence. As the inclusions of HPC $50 \%$ form filaments we also observe small droplets of PDMS coalescing to form filaments In droplets of HPC50\%, one or two encapsulated drops of PDMS can generally be observed. These mechanisms are shown schematically in Fig. 5(a).

(ii) In the stripes where the HPC50\% is continuous, filament and inclusions of PDMS break to form small droplets. Although it is not easy to measure it by optical microscopy, their size distribution seems to be very narrow compared with the size distribution of the inclusions of HPC $50 \%$ in the stripes of PDMS. This could be due to an absence of coalescence of PDMS droplets in these stripes.

(iii) At the boundary between PDMS and HPC50\% stripes, several complex mechanisms compete.

Droplets of PDMS dispersed in the matrix of HPC $50 \%$ can coalesce with the stripes of PDMS and contribute, in this way, to the broadening of the PDMS stripes.

The existence of a filamen of HPC50\% at the edge of a stripe of PDMS can lead to two opposite mechanisms.

The layer of PDMS between the filamen of HPC $50 \%$ and the stripe of $\mathrm{HPC} 50 \%$ can also constitute a filament Depending on the breakage time of this filamen (whether it is 
shorter or longer than that of HPC50\%), the stripe of PDMS will broader or become thinner. In the case of the development of Rayleigh instabilities in the thread of PDMS, the drops created will disperse in the stripe of HPC $50 \%$. The filamen of HPC $50 \%$ joins the continuous phase of HPC50\% and the subinclusions of PDMS encapsulated in this filamen are thus dispersed in the stripe of HPC $50 \%$ too. This mechanism leads to the broadening of the stripes of HPC50\% and so to the phase inversion. It is schematized in Fig. 5(b). If the breakage time of the filamen of HPC $50 \%$ is shorter than that of the filamen formed by the layer of PDMS, most of the droplets of HPC $50 \%$ created will contain subinclusions of PDMS. This mechanism leads to the broadening of the stripes of PDMS and to the refinement of the initial morphology. This mechanism is schematized in Fig. 5(c).

After such long shearing times, two different equilibrium morphologies can thus be obtained. In some cases, due to the thinning of the stripes of PDMS, the local phase inversion propagates into the whole sample [Figs. 4(c) and 4(d)]. Consequently, a general flow-induce phase inversion occurs. In other cases, the stripes of HPC $50 \%$ thin and disappear. The flow-induce phase inversion does not take place. As can be seen in Fig. 4(e), the resulting fina morphology is in this case a continuous phase of PDMS with filament and droplets of HPC50\% that themselves contain subinclusions of PDMS.

We did not note any influenc of the radial gradient of the shear rate over the width of visualization during these experiments. Indeed the formation and the broadening of the different stripes did not occur preferentially at one side of the window.

Some experiments were performed at higher gaps (up to $500 \mu \mathrm{m}$ ) to check the weak influenc of the gap. We observed with less sharpness the morphologies of the blends but their evolution was similar as for small gaps $(50$ or $100 \mu \mathrm{m})$. This was confirme once the shear was stopped at different stages, and the gap was slowly decreased up to the point where it was possible to observe the morphology distinctly. This is not direct in situ experiments, but interrupted ones.

\section{Evolution of the final morphology after cessation of flow}

Once the fina morphology was reached, the shear was stopped. For the blends where the phase inversion did not occur, a morphology similar to the initial morphology before shearing, but much finer develops a few minutes after cessation of flow The PDMS is the continuous phase, and the dispersed phase is composed of droplets of HPC $50 \%$ that encapsulate between one and three droplets of PDMS. This morphology is similar to the morphology of high-impact polystyrene.

If the flo is stopped after a phase inversion, a completely opposite morphology is observed. Immediately after stopping, the shear-deformed droplets of PDMS relax into spherical drops. The drops of PDMS coalesce but remain in the dispersed phase. In order to confir the stability of the inverted morphology (blends with HPC $50 \%$ continuous and PDMS dispersed) and the nonreversibility of the phase inversion, these blends were resheared 2 min after cessation of flo at $0.2 \mathrm{~s}^{-1}$, a shear rate where PDMS should be the continuous phase. But even after $15 \mathrm{~h}$ of shear, the HPC $50 \%$ was still the continuous phase as shown in Fig. 4(f).

\section{B. Influence of polymer concentration and shear rate on the final steady- state morphology}

At $10 \mathrm{~s}^{-1}$, whether a phase inversion is induced or not depends on concentration. As shown in Fig. 6, the occurrence of phase inversions observed during the tests at a given concentration increases with the concentration of HPC $50 \%$. With $60 \%$ of PDMS, the 


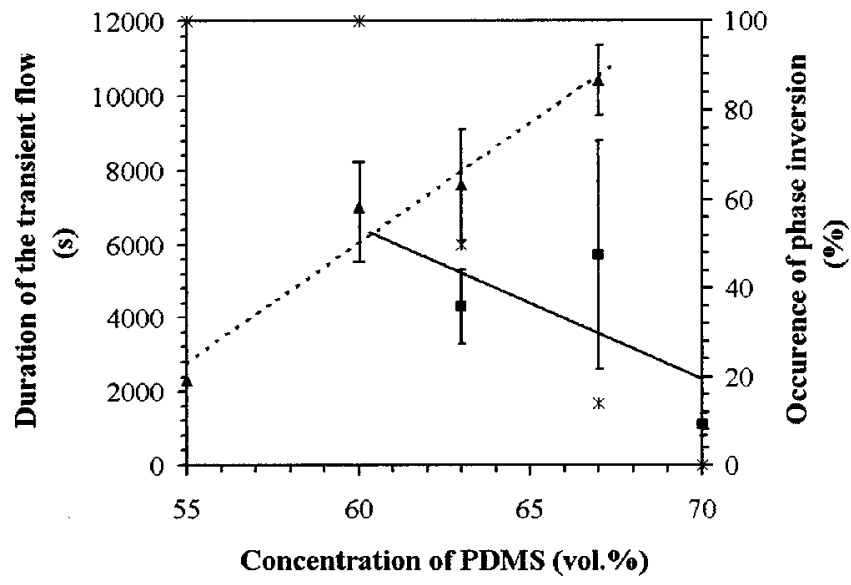

FIG. 6. Blends sheared at $10 \mathrm{~s}^{-1}$. Occurrence of phase inversion $\left(^{*}\right)$ vs concentration of PDMS. Duration of the transient flo $t_{3}$ vs concentration of PDMS in case of phase inversion $(\boldsymbol{\Delta})$ and refinemen $(\mathbf{\square})$. The lines $(---)$ and $(-)$ are linear fit of the experimental points.

phase inversion is always observed. With $70 \%$ of PDMS, a phase inversion is never induced. Between these two limits, there is a range of concentrations where, for the same blend, we obtain in some cases the inverted morphology after shearing and in other cases not. The HPC50\% viscosity being very sensitive to temperature, small variations could modify the viscosity ratio. Furthermore because the initial morphologies are not well controlled for the reasons discussed above, small differences in the at-rest morphologies (size of the inclusions, volume of PDMS encapsulated or small variations of concentrations) could influenc the fina morphology after shearing at $10 \mathrm{~s}^{-1}$ near the region of phase inversion. All these parameter variations could be sufficien to shift the system into or out of the region of phase inversion.

As co-continuity was observed for a range of concentrations instead of a single point of phase inversion, the critical concentration for flow-induce phase inversion cannot be determined by one point. There is rather a range of concentration for the blend to "hesitate" between both morphologies. Nevertheless, without extraction experiments we cannot confir that the fina steady-state morphology is passing through a transient cocontinuous morphology.

\section{Comparison with models based on viscosity ratio and elasticity ratio}

Our observations can be compared with the different models presented in the literature. From Eqs. (1), (2), and (5), the viscosity ratio necessary to have $\mathrm{HPC} 50 \%$ as the continuous phase can be calculated for each concentration. The minimal shear rate to apply for having a continuous phase of HPC $50 \%$ can be inferred from the rheological data in steady state [see Fig. 1(a)]. But since in the absence of interfacial slip the stresses are continuous across the interface while the deformation rates are not, the models have in common the fact that they consider only the viscosity ratio should be used at constant stress as reported by Utracki (1991). Calculations based on constant stress of one of the two phases give similar results as made at constant shear rate for the models of Miles and Zurek [Eq. (1)] and Metelkin and Blekht [Eq. (2)] (see Table II). Data calculated with the model of Utracki [Eq. (5)] depend on the way the viscosity ratio is calculated and cannot be compared with our experimental results. In the case of Bourry and Favis [Eq. (6)], the 
TABLE II. Minimal shear rate to apply to have HPC $50 \%$ as the continuous phase determined with the models of Miles and Zurek [Eq. (1)], Metelkin and Blekht [Eq. (2)], Utracki [Eq. (5)], and Willemse et al. [Eq. (7)] for blends of different concentrations [(i) calculated at constant shear rate; (ii) calculated at constant shear stress of HPC $50 \%$; (iii) calculated at constant shear stress of PDMS].

\begin{tabular}{|c|c|c|c|c|}
\hline \multirow[b]{2}{*}{ Blend } & \multicolumn{4}{|c|}{$\begin{array}{l}\text { Shear rate }\left(\mathrm{s}^{-1}\right) \text { calculated } \\
\text { with the different equations }\end{array}$} \\
\hline & [Eq. (1)] & [Eq. (2)] & [Eq. (5)] & {$[\text { Eq. (7) }]^{\mathrm{a}}$} \\
\hline \multicolumn{5}{|c|}{ PDMS55 } \\
\hline (i) & 1.8 & 1.7 & 3.1 & \\
\hline (ii) & 1.8 & 1.7 & 2.9 & 7.5 \\
\hline (iii) & 1.5 & 1.2 & 1.8 & \\
\hline \multicolumn{5}{|l|}{ PDMS60 } \\
\hline (i) & 2.2 & 1.7 & 7 & \\
\hline (ii) & 2.2 & 1.7 & 5.5 & 7.8 \\
\hline (iii) & 1.6 & 1.2 & 2.1 & \\
\hline \multicolumn{5}{|l|}{ PDMS63 } \\
\hline (i) & 3.1 & 1.7 & No & \\
\hline (ii) & 2.9 & 1.7 & 9 & 8.5 \\
\hline (iii) & 1.8 & 1.2 & 2.5 & \\
\hline \multicolumn{5}{|c|}{ PDMS67 } \\
\hline (i) & 4.6 & 1.8 & No & \\
\hline (ii) & 4 & 1.8 & 13 & 10 \\
\hline (iii) & 2 & 1.5 & 2.8 & \\
\hline \multicolumn{5}{|l|}{ PDMS70 } \\
\hline (i) & 7 & 1.8 & No & \\
\hline (ii) & 5.5 & 1.8 & 30 & 14 \\
\hline (iii) & 2.1 & 1.5 & 31 & \\
\hline
\end{tabular}

${ }^{\mathrm{a}}$ Fitted at $10 \mathrm{~s}^{-1}$ with our experimental results, using $R_{0}=23 \mu \mathrm{m}$.

necessity to know $G^{\prime}$ and $G^{\prime \prime}$ at constant shear stress (that we did not measure) does not allow us to perform such a comparison.

At $10 \mathrm{~s}^{-1}$, PDMS and HPC50\% have about the same elasticity (see Table I), which strongly simplifie evaluation of the influenc of viscosity and elasticity on the point of phase inversion. Nevertheless, the models of Miles and Zurek [Eq. (1)] and Metelkin and Blekht [Eq. (2)] have limits. For shear rates above $7 \mathrm{~s}^{-1}$, they predict a phase inversion on all of our blends. However, refinemen is observed for some blends with $63 \%$ of PDMS or more.

For shear rates below unity, the elasticity ratio and viscosity ratio are greater than unity. They should have opposite effects on the morphology. In order to test the influenc of elasticity on the fina morphology, a blend of PDMS63 was sheared at $0.8 \mathrm{~s}^{-1}$ (viscosity ratio $=1$ ) for $30 \mathrm{~h}$ (i.e., 86400 strain units). After a few hours, the stripe morphology appeared and no more evolution of the morphology was observed during the following $20 \mathrm{~h}$ of shearing. We did not get a phase inversion, as predicted by Eqs. (1) and (2) that nevertheless do not take into account the elasticity ratio.

Between 10 and $30 \mathrm{~s}^{-1}$, the elasticity ratio changes with the shear rate (from 1 to 0.5 ) while the viscosity ratio remains at a constant value of 0.4 . By increasing the shear rate and so decreasing the elasticity ratio, elasticity should limit the phase inversion. On the contrary, we observed with PDMS63 blends either phase inversion or refinemen with even a slight increase of the rate of occurrence of phase inversion (Fig. 7). 


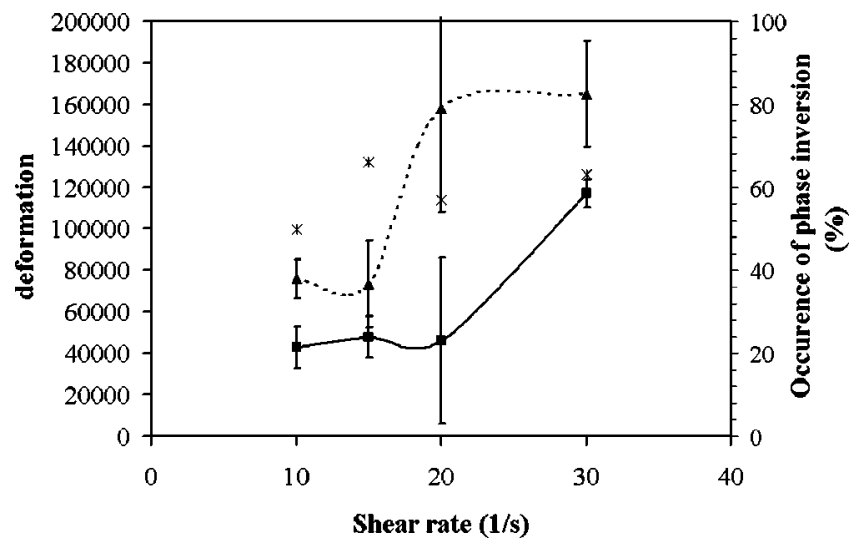

FIG. 7. Deformation in strain units necessary to reach either the refine (- - ) or the phase inverted morphology (-- $\mathbf{\Delta - - )}$ and frequency of occurrence $\left(^{*}\right)$ of phase inversion vs shear rate for the blend PDMS63.

The elasticity ratio does not seem to influenc the concentration of shear-induced phase inversion for elasticity ratios between 0.5 and 10 . But we have clear experimental limitations for exploring higher elasticity differences.

\section{Comparison with the model of Willemse et al.}

Although the model of Willemse et al. [Eq. (7)] presents the disadvantage of having the phase dimension as an unknown parameter, it has the advantage of taking into account more mixing parameters such as viscosity of the major phase, shear rate, and interfacial tension, which are well define in our experiments. Contrary to the other models, this model does not allow us to predict a point of phase inversion but a minimal concentration so that the initial dispersed phase becomes a continuous phase. Equation (7) was used to predict the effects of concentration or shear rate by using the phase dimension $R_{0}$ as a ft parameter.

At $10 \mathrm{~s}^{-1}$, the minimal concentration to have $\mathrm{HPC} 50 \%$ as a continuous phase is experimentally known: phase inversion is observed with a minimal concentration of HPC $50 \%$ of $33 \%$ (blend PDMS67). Knowing the shear rate, concentration of HPC $50 \%$ ( $\Phi_{\mathrm{HPC} 50 \%, \mathrm{cc}}$ ), matrix viscosity and interfacial tension $R_{0}$ can be calculated with Eq. (7). This is justifie because the phase dimension $R_{0}$ only changes during the initial stage of mixing. By supposing that the phase dimension is slightly influence by concentration on this concentration range, the critical shear rates for phase inversion can be calculated for the different concentrations. The results are presented in Table II (with $R_{0 \mathrm{HPC} 50 \%}$ $=23 \mu \mathrm{m}$ for PDMS67).

Ghodgaonkar and Sundararaj (1996) proposed a formulation to predict $D$, the dispersed phase drop diameter in viscoelastic blends

$$
D=\frac{2 \sigma}{\eta_{m} \dot{\gamma}-2\left(G_{d}^{\prime}-G_{m}^{\prime}\right)}
$$

with $G_{d}^{\prime}$ and $G_{m}^{\prime}$ the storage moduli of the dispersed and matrix phases. If we calculate $D$ with HPC50\% as the dispersed phase and PDMS as the matrix, we do not fin major differences in the range of shear rates calculated with Eq. (7) (see Fig. 8). This can justify the use of a constant phase dimension $R_{0}$ in Table II. Because of the qualitative deter- 


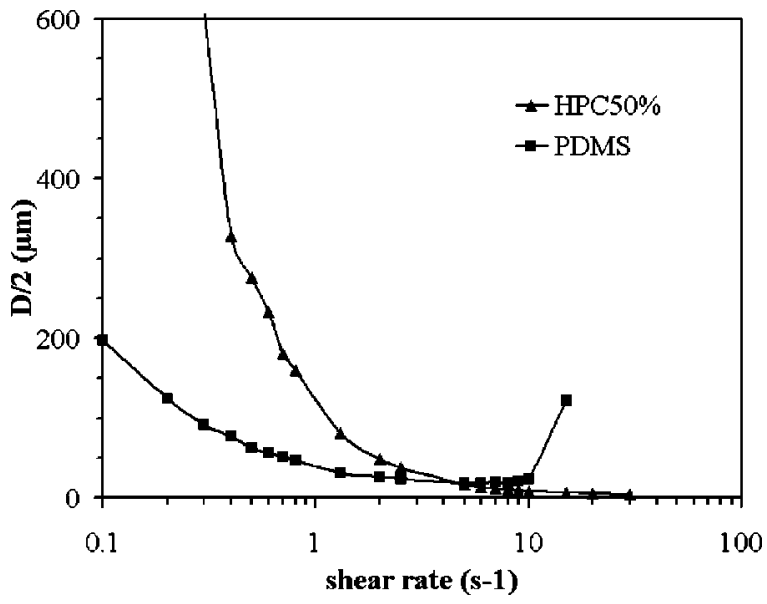

FIG. 8. Radius of the dispersed phase versus shear rate calculated with Eq. (8) proposed by Ghodgaonkar and Sundararaj (1996).

mination of $\Phi_{\mathrm{HPC} 50 \%, \mathrm{cc}}$ and $R_{0}$, these results do not allow us to determine quantitative values but to highlight a trend: the minimal shear rate to apply for having HPC $50 \%$ as the continuous phase increases with concentration of PDMS.

By reversing the role of PDMS and HPC50\% in Eq. (7), the apparent stability of the inverted morphology (blends with HPC50\% continuous and PDMS dispersed) and the nonreversibility of the phase inversion at low shear rates can be understood. We calculated at $0.2,0.8$, and $10 \mathrm{~s}^{-1}$ the minimal phase dimension $R_{0}$ of PDMS necessary to return as the continuous phase. With $67 \%$ of PDMS, $R_{0}$ of PDMS should be larger than $263 \mu \mathrm{m}$ at $0.2 \mathrm{~s}^{-1}, 108 \mu \mathrm{m}$ at $0.8 \mathrm{~s}^{-1}$, and $46 \mu \mathrm{m}$ at $10 \mathrm{~s}^{-1}$ to return as the continuous phase. This is much larger than what is experimentally observed at $0.2 \mathrm{~s}^{-1}$ [Fig. 4(f)] and $10 \mathrm{~s}^{-1}$ [Fig. 4(d)] or calculated with Eq. (8) (see Fig. 8).

\section{Phase change dynamics: Time necessary to reach the steady-state morphology}

The existing models give no information about the duration of the flo before the occurrence of phase inversion. Lazo and Scott (1999) made the assumption that the relevant parameter in the kinetics of phase inversion of PS/PE blends is the rate of hole nucleation in the forming sheets. But the sheets are formed with the least viscous component. They are so more easily stretched than if they were formed with the most viscous component. Phase inversion occurs after 1000-3500 strain units. In our case, the sheets of PDMS seem to be more stable. Our observations have shown that the time necessary to reach the inverted morphology is very long. The mechanisms leading to the fina steady-state morphology seem to occur at the edge of the sheets and not on their whole surface as proposed by this hole nucleation theory. Furthermore, it does not explain the case of refinement This mechanism is certainly present but not prevalent in our blends.

We can attribute a characteristic time to the different identifie transient stages of the flow For each type of blend, measurements were carried out for at least four or fiv samples. We defin $t_{0}$ as the start of shearing, $t_{1}$ as the time when the large domains of PDMS emerge (equivalent to the beginning of the second stage of morphology as described in the preceding paragraph), $t_{2}$ as the beginning of the stripe morphology (equivalent to the beginning of the fourth stage of morphology) and $t_{3}$ as the time 
TABLE III. Characteristic times of the three identifie steps to reach the fina steady-state morphology, for blends of different concentrations sheared at $10 \mathrm{~s}^{-1}$.

\begin{tabular}{cccccc}
\hline \hline $\begin{array}{c}\text { Final } \\
\text { steady-state } \\
\text { morphology }\end{array}$ & Blend & \multicolumn{1}{c}{$\begin{array}{c}t_{1} \\
(\mathrm{~s})\end{array}$} & $\begin{array}{c}t_{2} \\
(\mathrm{~s})\end{array}$ & \multicolumn{1}{c}{$\begin{array}{c}t_{3} \\
(\mathrm{~s})\end{array}$} & $\begin{array}{c}\dot{\gamma} t_{3} \\
\text { (strain units) }\end{array}$ \\
\hline Phase inversion & PDMS60 & 23 & 310 & 7000 & 70000 \\
& PDMS63 & 47 & 160 & 7600 & 76000 \\
& PDMS67 & 60 & 260 & 10400 & 104000 \\
Refinemen & & & & 4300 & 43000 \\
& PDMS63 & 54 & 180 & 5700 & 57000 \\
& PDMS67 & 330 & 910 & 1100 & 11000 \\
\hline \hline
\end{tabular}

necessary to reach the steady-state morphology (equivalent to the end of the fourth state of morphology). The boundary between the different stages is not very sharp, especially between the second and third stage. Actually, formation of the large domains of PDMS can continue while others are already being stretched to form stripes. Furthermore, it is difficul to determine the end of the last stage for the blends for which the inverted morphology does not occur. By contrast, for the blends that invert their morphology, we can easily determine the time $t_{3}$ when the last filamen of PDMS breaks up in the matrix of HPC $50 \%$. The results are given in Tables III and IV. It is clear that most of the time necessary to reach the steady-state morphology (refinemen or phase inversion) is devoted to the $t_{3}$ process. Compared to $t_{2}$ and $t_{3}$, the time necessary to obtain the bifibrilla morphology is very short, being nevertheless a few hundred strain units. The time $t_{2}$ necessary for obtaining the stripe morphology is much larger, and the corresponding deformation is a few thousands strain units. The limiting process is the interplay between coalescence and breakup that occurs during the fourth stage. It is also during this stage that the system will either refin its morphology or phase invert. The description of the phase inversion must thus take into account this stage. The other stages can be ignored as a firs approximation. We will now go into more details on the influenc of concentration and shear rate on the transition to the fina morphology.

TABLE IV. Characteristic times and deformation necessary to reach the steady-state morphology at different shear rates, for the blend PDMS63.

\begin{tabular}{cccccccr}
\hline \hline $\begin{array}{c}\text { Final } \\
\text { steady-state } \\
\text { morphology }\end{array}$ & $\begin{array}{c}\dot{\gamma} \\
\left(\mathrm{s}^{-1}\right)\end{array}$ & $\begin{array}{c}t_{1} \\
(\mathrm{~s})\end{array}$ & $\dot{\gamma} t_{1}$ & \multicolumn{1}{c}{$\begin{array}{c}t_{2} \\
(\mathrm{~s})\end{array}$} & $\dot{\gamma} t_{2}$ & $\begin{array}{c}t_{3} \\
(\mathrm{~s})\end{array}$ & $\dot{\gamma}_{3}$ \\
\hline Phase inversion & 10 & 47 & 470 & 160 & 1600 & 7600 & 76000 \\
& 15 & 32 & 480 & 90 & 1350 & 4900 & 73500 \\
& 20 & 11 & 220 & 440 & 8800 & 7900 & 158000 \\
& 30 & 23 & 690 & 100 & 3000 & 5500 & 165000 \\
Refinemen & 10 & 53 & 530 & 140 & 1400 & 7300 & 73000 \\
& 15 & 24 & 360 & 116 & 1700 & 3200 & 48000 \\
& 20 & 35 & 700 & 120 & 2400 & 3800 & 76000 \\
& 30 & 29 & 870 & 88 & 2640 & 2700 & 81000 \\
\hline \hline
\end{tabular}




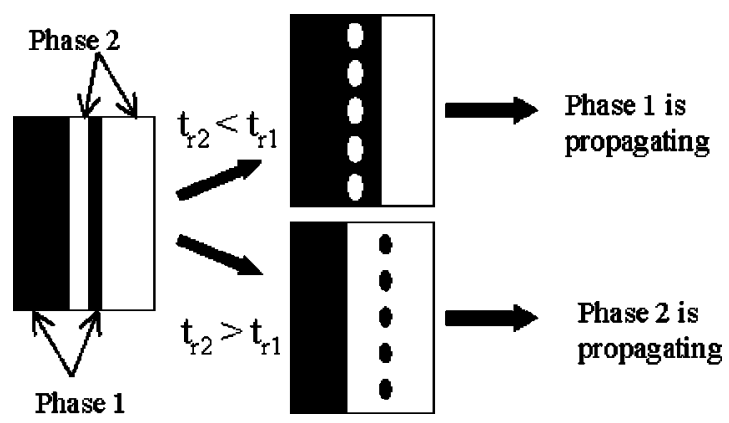

FIG. 9. Schematized mechanisms of breakage of one filamen occurring at the boundary of the stripes that leads to the propagation of one phase.

\section{Influence of the concentration at a shear rate of $10 \mathrm{~s}^{-1}$}

We recorded large discrepancies (up to 20\%) in characteristic times between samples at the same concentration. These discrepancies can be explained by the different initial morphologies. The size of the inclusions of $\mathrm{HPC} 50 \%$, and the size and especially the volume of encapsulated droplets of PDMS can be slightly different from one sample to another. The presence and the total volume of encapsulations of PDMS in the sample probably influence the duration of the mechanism of phase inversion. For samples of the same concentration, the phase inversion occurs earlier in blends containing a larger volume of encapsulated PDMS. Considering the experimental variability due to the different initial morphologies and the difficult of identifying $t_{1}$ and $t_{2}$, it is difficul to measure very precisely the influenc of concentration on $t_{1}$ and $t_{2}$. On the other hand, its influenc on $t_{3}$ can easily be measured.

The results for $t_{1}, t_{2}$, and $t_{3}$ are reported in Table III. Only $t_{3}$ is plotted in Fig. 6. It is important to observe how, in term of $t_{3}$, concentration has opposite effects on phase inversion and refinement Below a concentration of $60 \%$, only phase inversion occurs. Increasing the concentration increases the time taken for phase inversion. The time taken follows the reverse trend, decreasing with increasing concentration. At $63 \%$, refinement are sometimes obtained. At $70 \%$, we observe only refinemen as the steady-state morphology.

If we fi the two sets of points in Fig. 6 with straight lines, it is interesting to note that the two slopes have similar absolute values. This may indicate that these phenomena are similarly proportional to concentration, in this range of concentration. This makes sense if we recall that both steady-state morphologies are reached through the same mechanism of breaking fiber as schematized in Fig. 9. By making the assumption that the relevant parameter is $t_{b}$, the time of breakage of the filament at the boundary of the stripes, $t_{3}$ will be the number of filament which must be broken multiplied by the time of breakage of one filament

$$
\begin{gathered}
t_{3}=n_{\mathrm{HPC} 50 \%} \cdot t_{b \mathrm{HPC} 50 \%}<n_{\mathrm{PDMS}} \cdot t_{b \mathrm{PDMS}} \quad \text { in the case of refinemen } \\
t_{3}=n_{\mathrm{PDMS}} \cdot t_{b \mathrm{PDMS}}<n_{\mathrm{HPC} 50 \%} \cdot t_{b \mathrm{HPC} 50 \%} \quad \text { in the case of phase inversion, }
\end{gathered}
$$

where $n_{\mathrm{HPC} 50 \%}$ and $n_{\mathrm{PDMS}}$ are, respectively, the average number of filament of HPC $50 \%$ per stripe of PDMS and the average number of filament of PDMS per stripe of $\mathrm{HPC} 50 \%$ and $t_{b \mathrm{HPC} 50 \%}$ and $t_{b \text { PDMS }}$, respectively, the time of breakage of one filamen 
of HPC $50 \%$ and one filamen of PDMS. Tsakalos et al. (1998) estimated $t_{b}$, the time necessary for a drop to break through the development of capillary instabilities in blends of PDMS/HPC50\%

$$
t_{b}=\frac{1}{\dot{\gamma}} \cdot\left(\frac{D_{0}}{d_{b}(\dot{\gamma})}\right)^{2}
$$

with

$$
d_{b} \propto \frac{1}{\dot{\gamma}},
$$

where $D_{0}$ is the initial diameter of the drop before shearing and $d_{b}(\dot{\gamma})$ the thread diameter necessary for capillary instabilities leading to breakup. By supposing that

$$
\begin{gathered}
n_{\mathrm{HPC} 50 \%} \propto\left(1-\Phi_{\mathrm{PDMS}}\right) \\
n_{\mathrm{PDMS}} \propto \Phi_{\mathrm{PDMS}}
\end{gathered}
$$

and that $d_{b}$ is independent of concentration [Tsakalos et al. (1998)]:

$$
\begin{aligned}
& t_{3} \propto\left(1-\Phi_{\mathrm{PDMS}}\right) \cdot A(\dot{\gamma}) \quad \text { in the case of refinemen } \\
& t_{3} \propto \Phi_{\mathrm{PDMS}} \cdot B(\dot{\gamma}) \quad \text { in the case of phase inversion, }
\end{aligned}
$$

where $A(\dot{\gamma})$ and $B(\dot{\gamma})$ are the terms describing dependence on the shear rate. At a given shear rate, the time to obtain a given phase as the continuous phase should be proportional to the concentration of this phase. This is what we observe in Fig. 6.

\section{Influence of the shear rate}

PDMS63 blends were sheared at various shear rates above $10 \mathrm{~s}^{-1}$, i.e., 15,20 , and 30 $\mathrm{s}^{-1}$. As shown in Table I, the viscosity ratio is the same at these shear rates, but not the elasticity ratio. Increasing the shear rate decreases $G_{\mathrm{HPC} 50 \%}^{\prime} / G_{\mathrm{PDMS}}^{\prime}$ by a factor 2 at 30 $\mathrm{s}^{-1}$ (compared to $G_{\mathrm{HPC} 50 \%}^{\prime} / G_{\mathrm{PDMS}}^{\prime}=1$ at $10 \mathrm{~s}^{-1}$ ).

Figure 7 shows that the amount of deformation necessary to reach equilibrium is an apparently nonmonotonically function of the shear rate. Deformation versus shear rate for refinemen and phase inversion both increase with shear rate, but not at the same rate. Once more, we see here that since the same mechanisms are responsible for both morphologies, external parameters have the same influenc on both morphologies. The question remains whether to consider the plateau of deformation between 10 and $20 \mathrm{~s}^{-1}$ for refinemen and between 20 and $30 \mathrm{~s}^{-1}$ for phase inversion as real, or as being simply due to the difficult of measuring when the steady state is really reached. The only relevant parameters acting in the process are breakup and coalescence, which are monotonically dependent on the shear rate. In addition, the viscosity ratio is constant and the elasticity ratio varies monotonically between 10 and $30 \mathrm{~s}^{-1}$.

If we consider the breakage of filament as the relevant process, it is found from Eqs. (10) and (11) that $t_{b}$ is proportional to $\dot{\gamma}$. Also, at a given concentration, the deformation for having one phase to be the continuous one should be

$$
\text { deformation }=\dot{\gamma} \cdot t_{3}\left\{\begin{array}{l}
\propto \dot{\gamma}^{2} \cdot C\left(1-\Phi_{\mathrm{PDMS}}\right) \quad \text { in the case of refinemen } \\
\propto \dot{\gamma}^{2} \cdot D\left(\Phi_{\mathrm{PDMS}}\right) \quad \text { in the case of phase inversion }
\end{array}\right.
$$

where $C\left(1-\Phi_{\mathrm{PDMS}}\right)$ and $D\left(\Phi_{\mathrm{PDMS}}\right)$ regroup terms dependent on concentration. At a given concentration of $63 \mathrm{vol} \%$, this is not what we observe experimentally (Fig. 7). This 
demonstrates the limits of this simplifie model. The equations for the time necessary for a drop to break [Eq. (10)] and the critical diameter of the filamen for breakup [Eq. (11)] proposed by Tsakalos et al. (1998) have been established for isolated droplets and so do not take into account the influenc of neighboring threads nor the influenc of coalescence. Nevertheless, for highly concentrated blends, the surrounding drops and threads probably have an effect on the stability on the filaments Furthermore, elasticity of the components has not been considered. But the decrease of $G_{\mathrm{HPC} 50 \%}^{\prime} / G_{\mathrm{PDMS}}^{\prime}$ with increasing shear rate certainly influence $d_{b}$ and $t_{b}$. Another question is which $D_{0}$ to consider in Eq. (10), especially in the case of $t_{b}$ PDMS where PDMS is the continuous phase at rest.

\section{CONCLUSIONS}

By varying the shear rate applied to blends of PDMS/HPC50\%, a flow-induce phase inversion can be observed and studied with rheo-optical microscopy. These observations show that phase inversion occurs after the blend passes through several different transient morphologies. A bifibrilla network structure appears during the transient flow as proposed by Luciani et al. (1993). But the subsequent transient stripe morphologies that follow the formation of sheets of PDMS demonstrate that the mechanisms leading to the inverted morphology are more complex. The blends must be subjected to larger deformations (strain units above 70000 ) than it was previously reported in a blend where the phase to disperse is the minor and least viscous component [Lazo and Scott (1999)]. In our system, the phase to disperse is the major and most viscous component. Fixing concentration and viscosity ratio, the amount of deformation necessary to reach the phase inversion is not constant and seems to depend on shear rate. This suggests that one of the controlling parameters is the amount of deformation needed to break a filament which is known to be shear rate dependent. At a fixe shear rate of $10 \mathrm{~s}^{-1}$, we did not observe a single clear concentration above which phase inversion would not occur. On the contrary, we rather observed a range of concentration for which either phase inversion or refine ment occurs. Again, if the rupture of the filament' mechanism is important, its dependence on the elasticity of the matrix and the filamen must be taken into account.

\section{References}

Avgeropoulos, G. N., F. C. Weissert, P. H. Biddison, and G. G. A. Böhm, "Heterogeneous blends of polymers. Rheology and morphology," Rubber Chem. Technol. 49, 93-104 (1976).

Bouilloux, A., B. Ernst, A. Lobbrecht, and R. Muller, "Rheological and morphological study of the phase inversion in reactive polymer blends," Polymer 38, 4775-4783 (1997).

Bourry, D. and B. D. Favis, "Co-continuity and phase inversion in HDPE/PS blends: influenc of interfacial modificatio and elasticity," J. Polym. Sci., Part B: Polym. Phys. 36, 1889-1899 (1998).

Chapoy, L. L., "The applicability of a percolation model to photoconductivity in guest-host thermotropic nematic liquid crystal systems," Chem. Phys. 84, 1530-1532 (1986).

Chappelear, D. C., "Interfacial tension between polymers," Polym. Prepr. (Am. Chem. Soc. Div. Polym. Chem.) 5, 363-371 (1964).

Favis, B. D. and J. P. Chalifoux, "Influenc of composition on the morphology of polypropylene/polycarbonate blends," Polymer 29, 1761-1767 (1988).

Ghodgaonkar, P. G. and U. Sundararaj, "Prediction of dispersed phase drop diameter in polymer blends: the effect of elasticity," Polym. Eng. Sci. 36, 1656-1665 (1996).

Guido, S. and N. Grizzuti, "Phase separation effects in the rheology of aqueous solutions of hydroxypropylcellulose," Rheol. Acta 34, 137-146 (1995).

Krieger, I. M. and T. J. Dougherty, "A mechanism for non-Newtonian flo in suspensions of rigid spheres," Trans. Soc. Rheol. 3, 137-152 (1959).

Lazo, N. D. B. and C. E. Scott, "Morphology development during phase inversion of a PS/PE blend in isothermal, steady-shear flow,' Polymer 40, 5469-5478 (1999). 


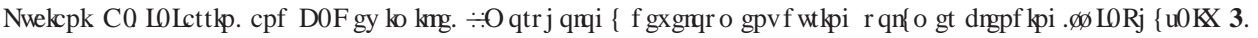

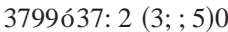

0 HNTCHI 1 I DQG + 9 HKRRJ W

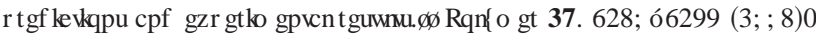

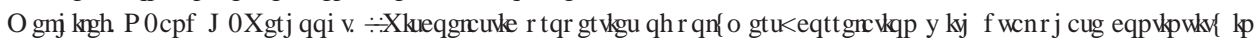

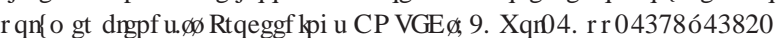

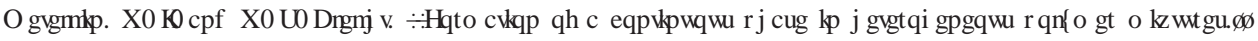

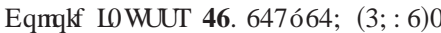

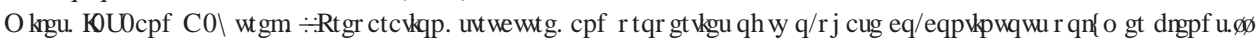

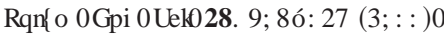

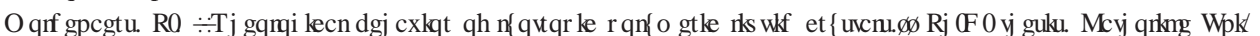

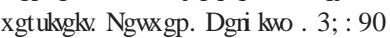

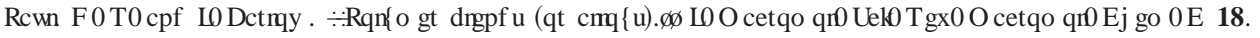

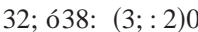

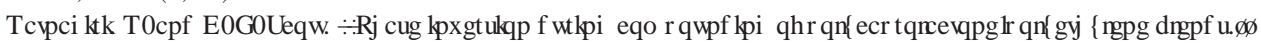

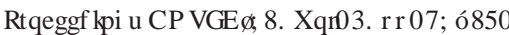

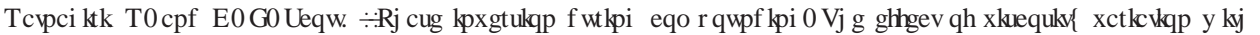

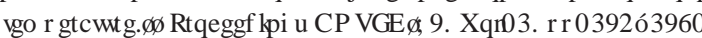

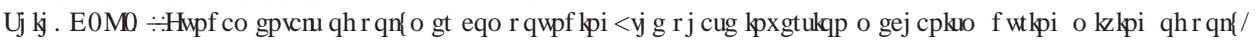

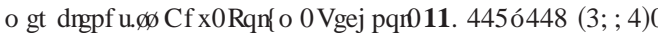

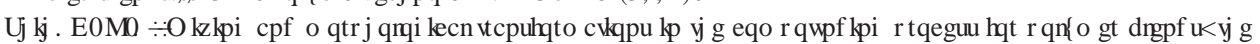

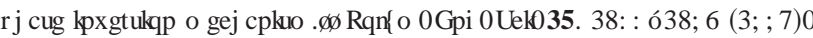

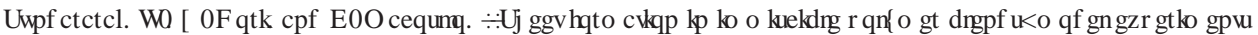

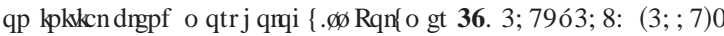

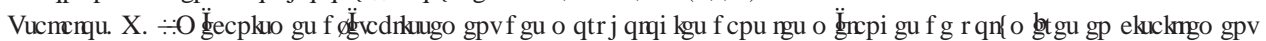

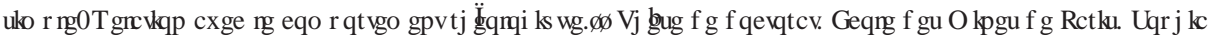

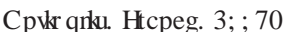

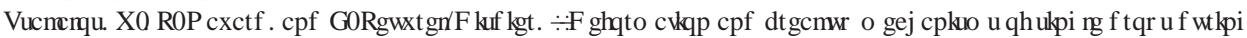

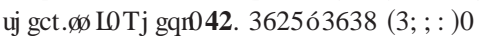

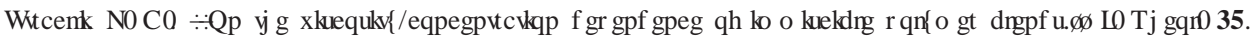

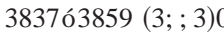

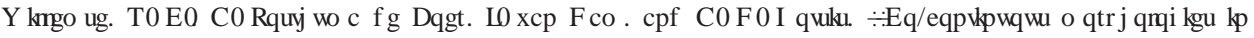

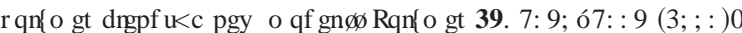

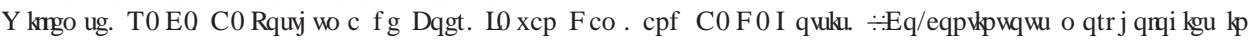

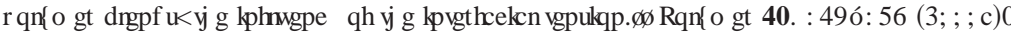

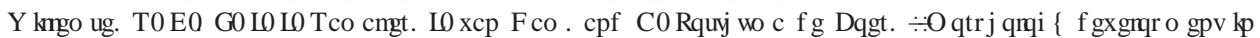

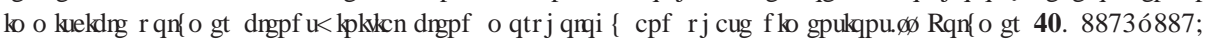
(प००स्ट) 